\title{
Spectral analysis and experimental study of lateral capillary dynamics for flip-chip applications
}

\author{
P. Lambert • M. Mastrangeli · J.-B. Valsamis • \\ G. Degrez
}

Received: 8 December 2009/ Accepted: 11 February 2010/Published online: 31 March 2010

(C) Springer-Verlag 2010

\begin{abstract}
This article presents a study on the dynamics of lateral motion of a liquid meniscus confined by a pad and a chip moving parallel to the pad. This problem is a typical flip-chip case study, whose use is widespread in industrial assembly. The proposed model describing this dynamics is built upon two coupled physics: the NavierStokes equation governing the liquid flow between the pad and the chip, and the Newton's law describing the motion of the chip. This coupled problem is solved with a spectral method based on Chebyshev polynomials, by assuming a linear analytical expression of the lateral stiffness of the meniscus in the cases of circular and square pads. The theoretical results are benchmarked with literature results and thoroughly experimentally validated. From these results, we propose a map giving the characteristic time of the chip dynamics according to only two non-dimensional parameters, constructed with
\end{abstract}

\section{P. Lambert ( $\square)$}

Automatic Control and Micro-Mechatronic Systems Department, FEMTO-ST Institute, UMR CNRS 6174 , UFC /ENSMM/ UTBM, 24 rue Alain Savary, 25000 Besançon, France

e-mail: pierre.lambert@femto-st.fr; pierre.lambert@ulb.ac.be

P. Lambert · J.-B. Valsamis

BEAMS Department, Université Libre de Bruxelles,

Avenue F. D. Roosevelt 50, CP 165/56, 1050 Bruxelles, Belgium

M. Mastrangeli

IMEC, Kapeldreef 75, 3001 Leuven, Belgium

M. Mastrangeli

MTM Department, Katholieke Universiteit Leuven, Kasteelpark Arenberg 44, 3001 Leuven, Belgium

G. Degrez

ATM Department, Université Libre de Bruxelles, Avenue F. D. Roosevelt 50, CP 165/43, 1050 Bruxelles, Belgium the physical (density, surface tension, and viscosity), geometrical (pad area and gap), or dynamical (chip mass) parameters of the problem.

Keywords Self-assembly $\cdot$ Flip-chip $\cdot$ Spectral method · Chebyshev $\cdot$ Surface tension · Damping

\section{Introduction}

Self-assembly processes for packaging applications are manyfold. Dry self-assembly (Park and Bohringer 2008), hydrophillic-hydrophobic interactions (Onoe et al. 2007) are being employed. Mastrangeli et al. (2009a) reviews shape matching, magnetic, electrophoretic, dielectrophoretic, and capillary mechanisms, the latter one falling into two categories. The first category is the fluid-driven capillary self-assembly, which has the advantage of accurate self-alignment, and the second category is the molten solder-driven self-assembly, which allows mechanical and electrical connections simultaneously. This article concerns both capillary mechanisms.

In the field of 2D positioning, Boufercha reported selfcentering by translation of components (Boufercha et al. 2008) while (Sariola et al. 2008; Tsai et al. 2007) reported on rotational self-positioning as well. Theoretically, these systems evolve toward minimum energy configurations which correspond to the desired positions, defined by the patterns on a substrate (see Mastrangeli et al. (2009b) for a study on patterned substrates coating for fluidic self-assembly). Unfortunately, imperfections such as, e.g., contact angle hysteresis lead to positioning errors. Therefore, Abbasi et al. (2008) proposed to add mechanical vibration to get loose of any local energy minimum and move toward the desired equilibrium 
position. Saeedi et al. reviewed the use of molten alloydriven self-assembly at nano- and micro-scale (Saeedi et al. 2006) in various application domains: forming metal contacts at nanoscale, 2D self-assembly, and also 3D self-assembly of freestanding components. Interestingly, they provide a database of physical properties of molten alloys such as surface tension and melting temperature. It is worth to note that the viscosity is not thereby mentioned; hence the characterization of the dynamics has not been taken into account. Many fluidic assembly case studies have been studied: micro-electro mechanical systems packaging (Fang 2006), 3D microopto-electro-mechanical systems [where, e.g., a mirror patterned in-plane is rotated out-of-plane thanks to surface tension actuated hinges (Hong and Syms 2006)], arrays of optical fibers onto an optical chip [with misalignment claimed to be in the order of $1 \mu \mathrm{m}$ (Avital and Zussman 2006)], RFID microchips (Tsai et al. 2007). Even biological samples such as drosophila embryos have been self-assembled on patterned sites (Zhang et al. 2005). According to (Zheng et al. 2004), self-assembly, based on geometrical shape recognition and subsequent binding to form mechanical and electrical connections, provides a route to non-robotic parallel assembly of electrical functional hybrid microsystems in three dimensions. Nevertheless, the core application of capillary self-assembly for this purpose comes from the pickand-place assembly of surface mounted devices (SMD) components, or from flip-chip. In pick-and-place, a component is placed on a solder paste deposited through a stencil on the substrate. During the reflow phase of the process, the solder is molten which, on the one hand, can provide the final positioning but, on the other hand, leads to misalignment due to a temporary loss of binding stiffness. In flip-chip, solder pasted bumps between two wafers ensure the electrical connections between one another: the final alignment of the wafers also requires the reflow phase, leading to similar issues.

Consequently, beside applications, the capillary selfassembly process needs to be (and has already been) modeled. This article reports on the modeling and experimental study of the dynamical lateral behavior of a liquid meniscus. Section 2 reviews existing models, and presents the coupled physical equations to be solved. Section 3 explains how to approximate the lateral stiffness of a meniscus in the cases of circular and square pads. Section 4 presents the solution of the coupled problem achieved by means of a spectral method based on Chebyshev polynomials. Experimental materials and methods are presented in Sect. 5, while results are described in Sect. 6. Finally, conclusions are drawn in Sect. 7.

\section{State of the art and definition of the problem}

Most modeling works are quasi-static, i.e., based on surface energy minimization to find the equilibrium positions of the system, and on energy gradient to compute restoring forces (Lin et al. 1995; Bohringer et al. 2004). For small displacements, an analytical model to estimate the lateral restoring force developed by a meniscus between two square pads is sketched in (Tsai et al. 2007) (see details in Sect. 3.3). Nevertheless, most of these studies does not address dynamical aspects such as characteristic damping time and resonance frequencies.

In this direction, van Veen (1999) derived analytical relations to model both the axial compressive motion and the lateral motion (Fig. 1). Kim et al. (2004) proposed a study on dynamic modeling for resin self-alignment mechanism. As the authors used a material with a low surface tension such as liquid resin, they claimed the alignment motion to be different from the oscillatory motion of the solder described by van Veen (1999). Recently, Lin et al. compared 2D numerical results with experiments (Lin et al. 2008). To solve these motion equations, these authors used the CFD-ACE+ package making use of an iterative algorithm alternating one time step resolution of Navier-Stokes equations (and the continuity equation) with one time step resolution of the structural mechanics equations (Lin et al. 2008). The authors observed a good match of their 2D simulation to experimental data in case of fluid meniscus aspect ratios larger than 3 .

Finally, Lu and Bailey (2005) predicted the dynamical behavior of a chip in flip-chip alignment, whose dynamics is governed by the following Newton's law:

$m \ddot{x}=-k(x) x-c \dot{x}$,

where $k(x)$ is the lateral stiffness of a meniscus linking a circular pad and a circular substrate, and the viscous force $-c \dot{x}$ is applied by the viscous stress on the chip. The lateral stiffness was computed with Surface Evolver and found to be almost constant, while the viscous force $c \dot{x}=\left.\eta S \frac{\partial u}{\partial y}\right|_{y=h}$ was computed from the liquid flow between the chip and the pad, governed by the Navier-Stokes equation $(\eta$ is the dynamic viscosity of the liquid, $S$ is the area of the chip in contact with liquid, $u$ is the velocity along $x$-axis, and $y$ is the coordinate perpendicular to the chip):

$\rho \frac{\partial u}{\partial t}-\eta \frac{\partial^{2} u}{\partial y^{2}}=0$

The coupled problem defined by Eqs. 1, 2 is thus described by two unknowns: first the position of the chip $x(t)$, and second the velocity profile inside the meniscus $u(y, t)$. 


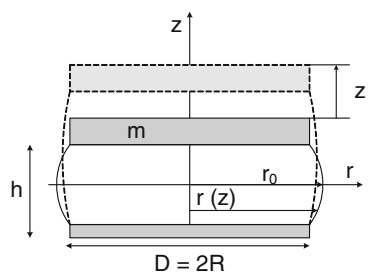

(a)

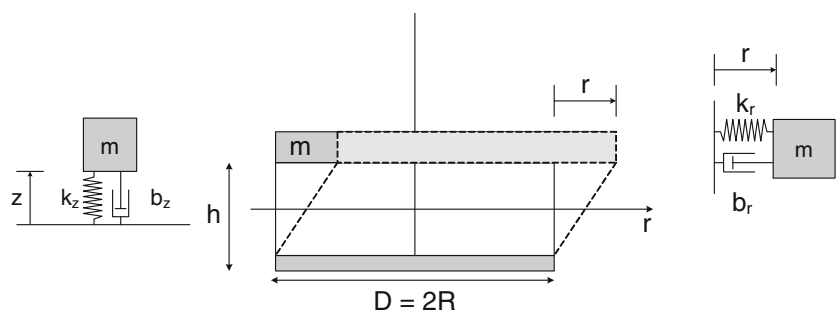

(b)

Fig. 1 Axial and radial models used by van Veen (1999). a Axial shift and b radial shift

The first equation (1) is associated with the following initial conditions ( $\delta$ is the initial elongation):

$x(t=0)=\delta$

$\dot{x}(t=0)=0$

while the initial and boundary conditions for the second equation (2) are:

$u(y=0, t)=0 \quad$ (no slip condition)

$u(y=h, t)=\dot{x}(t) \quad$ (coupling term)

$u(y, t=0)=0 \quad$ (zero initial velocity)

To decouple the equations of this problem, $\mathrm{Lu}$ and Bailey solved this problem by assuming a linear velocity profile $u(y, t)=(y / h) \dot{x}$. They also considered the application of a constant force along the top surface of the meniscus to compute the velocity profile. Their developments led to several response times: they showed that considering the chip dynamics only, the pseudo-period of the oscillating motion was of the order of $3.77 \mathrm{~ms}$ (using the underlying assumption of a linear velocity profile). On the other hand, they also showed that the characteristic time required for the velocity profile to become linear was of the same order of magnitude $(5.3 \mathrm{~ms})$. Therefore, they concluded that both physics (chip dynamics and fluid dynamics inside the meniscus) were strongly coupled and should be solved accordingly. They consequently proposed an iterative scheme including the alternate use of Surface Evolver to compute the meniscus stiffness and of a CFD package to compute the liquid flow.

To solve the same problem more efficiently, we hereby propose to use an analytical model for the lateral stiffness (Sect. 3) and a resolution based on Chebyshev polynomials, which transform the coupled problem of Eqs. 1, 2 into the form of a system of constant-coefficients ordinary differential equations (ODEs).

We finally mention the need to study the other degreesof-freedom (dof): beside the lateral motion, it is necessary to study the axial and tilt motions dynamically. Concerning the first one and additionally to the study of van Veen, partial information can be found in Engmann et al. (2005)
(Stefan equation to estimate the axial viscous force) Meurisse and Querry (2006) and Pascarella and Baldwin (1998) (the latter one studied the compression flow modeling). As far as the tilt motion is concerned, we refer to the work of Kaneda et al. (2007), where use is made of water and also glycerine $\left(\gamma=0.0635 \mathrm{~N} \mathrm{~m}^{-1}\right.$ and $\eta=$ $0.900 \mathrm{~Pa}$ s) to study the oscillation of a tilted circular pad on a droplet for the self-alignment process. With their model, these authors estimated the damping ratio and the oscillating frequencies.

\section{Lateral stiffness of liquid meniscus}

\subsection{General approach}

The equilibrium shape of a meniscus is ruled by the socalled Laplace equation (Lambert 2007) which in the axially symmetric case is written as a non-linear second-order differential equation. Except in the case of a cylindrical meniscus or in the case of the catenoid, this problem does not have analytical solutions. Nevertheless, analytical approximations can be found in the case of circular and square pads. These approximations can be benchmarked using numerical solutions obtained with Surface Evolver (Brakke 1992). It will be interesting to note that in both cases, the stiffness is proportional to half-perimeter of the wetted chip and to the surface tension, and inversely proportional to the gap. Another way to interpret this result is to note that the lateral stiffness is proportional to the ratio of the component size over the gap of the meniscus.

\subsection{Circular pads}

To estimate analytically the lateral stiffness of a meniscus confined between two circular pads, we compute first the lateral area of a tilted cylinder of height $h$, radius $R$, and shift $s$ (also called elongation $x$ in Fig. 2): this means that the cylinder axis is not perpendicular to both circles but inclined with an angle $\phi$ given by $\tan \phi=s / h$. 


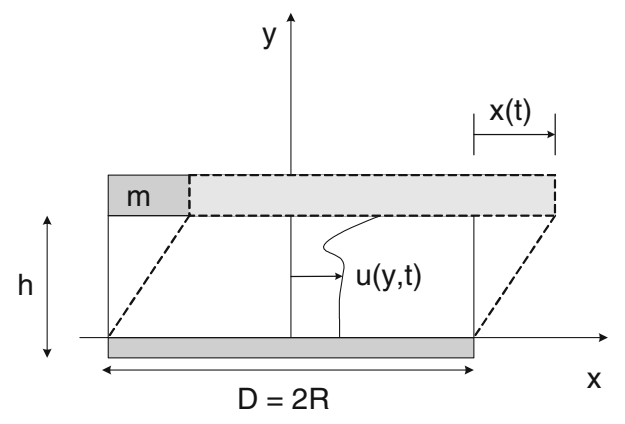

Fig. 2 Problem modeled in this work: a moving top pad is linked to a fixed bottom pad through a liquid meniscus whose velocity profile is assumed to be described by the horizontal velocity component $u$ only

The vectorial equation of a point $P$ of this cylinder is given by:

$$
\begin{aligned}
\overline{O P} & =z \overline{1}_{z}+s(z) \overline{1}_{x}+R \overline{1}_{r} \\
& =\left(\frac{z}{h} s+R \cos \theta\right) \overline{1}_{x}+(R \sin \theta) \overline{1}_{y}+z \overline{1}_{z}
\end{aligned}
$$

The lateral area is consequently equal to:

$$
S=R \int_{0}^{h} \mathrm{~d} z \int_{0}^{2 \pi} \sqrt{1+\cos ^{2} \theta \frac{s^{2}}{h^{2}}} \mathrm{~d} \theta
$$

and the total surface energy of the system is here equal to:

$E=\gamma S=\gamma R h \int_{0}^{2 \pi} \sqrt{1+\cos ^{2} \theta \frac{s^{2}}{h^{2}}} \mathrm{~d} \theta$,

where $\gamma$ is the surface tension.

The lateral restoring force is consequently equal to:

$F=-\frac{\partial E}{\partial s}=-\frac{\gamma R s}{h} \underbrace{\int_{0}^{2 \pi} \frac{\cos ^{2} \theta}{\sqrt{1+\cos ^{2} \theta \frac{s^{2}}{h^{2}}}} \mathrm{~d} \theta}_{I}$

The integral $I$ in the latter equation can be numerically computed (see the result in Fig. 3) or expressed in terms of elliptic integrals of the first (EllipticK) and second (EllipticE) kinds:

$I=4 \frac{h^{2}}{s^{2}}\left[\operatorname{EllipticE}\left(\sqrt{-\frac{s^{2}}{h^{2}}}\right)-\right.$ EllipticK $\left.\left(\sqrt{-\frac{s^{2}}{h^{2}}}\right)\right]$

Nevertheless, it is interesting to calculate it in our domain of interest (i.e., for small $s / h$ ratio, $\left.\sqrt{1+\cos ^{2} \theta\left(s^{2} / h^{2}\right.}\right) \approx 1$ in Eq. 11), which gives the following analytical relationship:

$F=-\frac{\partial E}{\partial s}=-\pi R \gamma \frac{s}{h}$ which corresponds to a constant stiffness $k_{\mathrm{c}}$ given by:

$k_{\mathrm{c}}=\frac{\pi R \gamma}{h}$

The existence of an analytical expression of the stiffness allows the numerical simulation presented in section 4 , where the advantages of such a resolution will be discussed.

This formulation has been benchmarked using Surface Evolver, in the case of $r=50 \mu \mathrm{m}, h=70 \mu \mathrm{m}, \quad \gamma=$ $0.325 \mathrm{~N} \mathrm{~m}^{-1}$ and a volume of liquid given by $V=\pi r^{2} h$. The comparison is plotted in Fig. 3. The discrepancy between the numerical and Surface Evolver results comes from the fact that the cylindrical geometry of Eq. 8 is not physically exact, since such a shape does not have a constant curvature.

\subsection{Square pads}

Tsai et al. (2007) already presented a model to compute the lateral stiffness of a square pad shifted by a distance $s$ along one of its edges. This model is based on the assumption of a prismatic meniscus whose volume is equal to the area of the square pad multiplied by the gap. In this case, a shift of the component keeps the volume constant at constant gap, but the lateral area $\Sigma$ of the liquid-vapor interface is increasing, given by:

$\Sigma=2 c h+2 c \sqrt{h^{2}+s^{2}}$

Consequently, the surface energy $E$ in this problem is given by:

$E=\gamma \Sigma+C$,

where $C$ is an arbitrary constant. The derivation with respect to the shift $s$ leads to the restoring force:

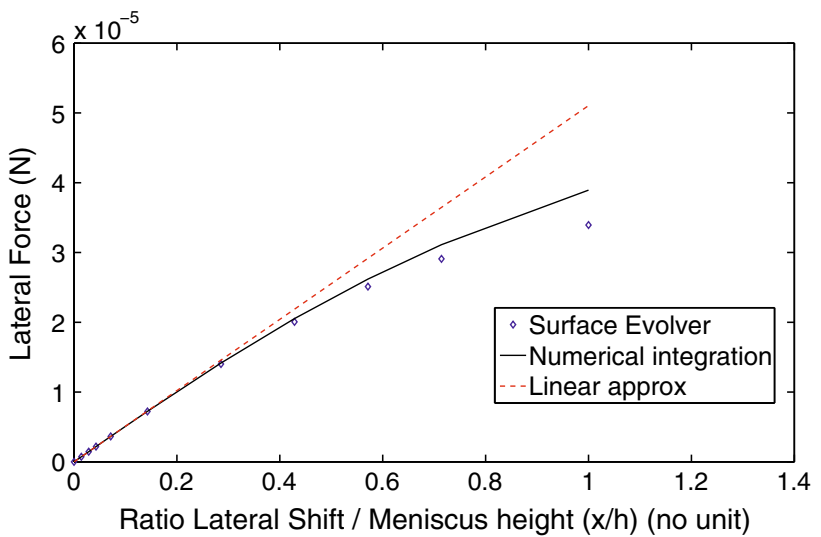

Fig. 3 Lateral restoring force and related stiffness of a cylindrical meniscus: comparison between the Surface Evolver benchmark, the analytical approximation of Eq. 13 and the numerical evaluation of Eq. $11\left(R=50 \mu \mathrm{m}, h=70 \mu \mathrm{m}\right.$ and $\left.\gamma=0.325 \mathrm{~N} \mathrm{~m}^{-1}\right)$. Note that the "_" sign of the force has been omitted here 
$F=-\frac{2 \gamma c s}{\sqrt{s^{2}+h^{2}}} \approx-\frac{2 c s \gamma}{h}$

The approximation is consistent with the assumption that $s<h$ and consequently $s^{2} \ll h^{2}$. To fix the ideas, let us consider $s<h / 10$. Note that the force direction is opposite to the shift. The force derivative leads to the stiffness:

$k_{\mathrm{s}}=\frac{2 c h^{2} \gamma}{\left(s^{2}+h^{2}\right)^{\frac{3}{2}}} \approx \frac{2 c \gamma}{h}$

Note well that $2 c$ in this formula is half perimeter of the pad, which was also the case in the circular pad $(\pi R \gamma / h)$. The comparison between Eq. 18 and Surface Evolver is shown in Fig. 5, where again there is a small difference between the model and Surface Evolver because the shape of the Fig. 4 does not have a constant curvature.

\section{Coupled problem resolution with a pseudo-spectral method based on Chebyshev polynomials}

The problem described by Eqs. 1, 2 is first normalized using characteristic length $\delta$ and time $\tau$ :

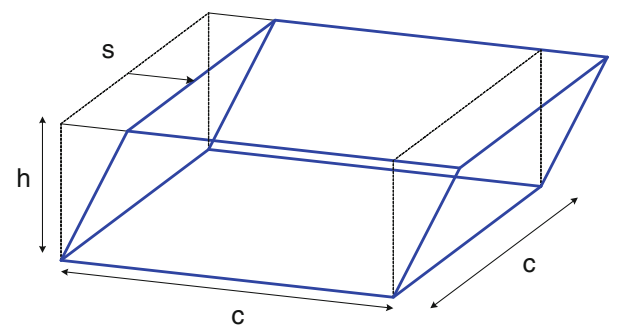

Fig. 4 Geometrical model used to compute the lateral stiffness of a square pad

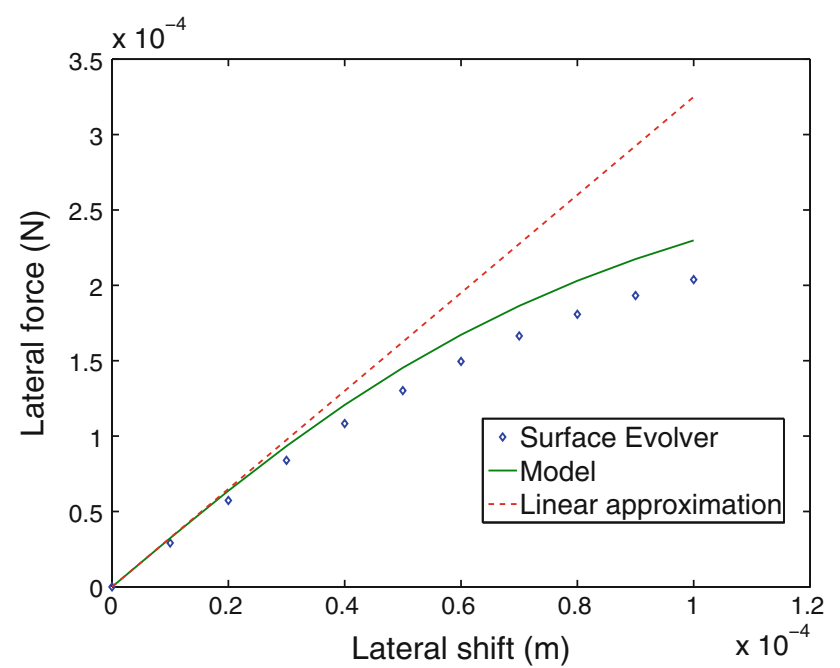

Fig. 5 Lateral capillary force in case of a square pad with edge $c=$ $500 \mu \mathrm{m}$, a gap $h=100 \mu \mathrm{m}$, a surface tension $325 \mathrm{mN} \mathrm{m}^{-1}$ $x=\delta \tilde{x}$

$t=\tau \tilde{t}$

$u=\frac{\delta}{\tau} \tilde{u}$,

where $\delta$ is the initial position of the chip and $\tau=\sqrt{\frac{m}{k}}$ is a characteristic time of the chip dynamics. The $y$ coordinate ranging from 0 to $h$ is replaced by $\tilde{y}$ ranging from -1 to 1 $\left(y=(1+\tilde{y}) \frac{h}{2}\right)$. This leads to two non-dimensional equations:

$\ddot{\tilde{x}}=-\tilde{x}-\left.\underbrace{\frac{\rho S h}{2 m}}_{\tilde{m}^{-1}} \underbrace{\frac{4 v \tau}{h^{2}}}_{\alpha} \frac{\partial \tilde{u}}{\partial \tilde{y}}\right|_{\tilde{y}=+1}$

$\frac{\partial \tilde{u}}{\partial \tilde{t}}=\frac{4 v \tau}{h^{2}} \frac{\partial^{2} \tilde{u}}{\partial \tilde{y}^{2}}$

with the following boundary and initial conditions:

$\tilde{u}(\tilde{y}=-1, \tilde{t})=0$

$\tilde{u}(\tilde{y}=+1, \tilde{t})=\dot{\tilde{x}}$

$\tilde{u}(\tilde{y}, \tilde{t}=0)=0$

$\tilde{x}(\tilde{t}=0)=1$

$\dot{\tilde{x}}(\tilde{t}=0)=0$

It can be seen from both non-dimensional equations that the problem only depends on two non-dimensional parameters: (i) $\alpha=4 v \tau / h^{2}$ the diffusion coefficient and (ii) $\tilde{m}=2 m /(\rho S h)$ the mass ratio.

In the following the symbol $\sim$ has been dropped everywhere (excepted for the mass ratio $\tilde{m}$ ) and the superscript ${ }^{(i)}$ indicates the $i$ th derivative with respect to $y$. With these conventions, the unknown velocity field $u$ can be written as a series of Chebyshev polynomials $T_{k}(y)$ :

$u(y, t)=\sum_{k=0}^{N} a_{k}(t) T_{k}(y)$,

where the series has been found in our case to converge toward $u(y, t)$ as soon as the order $N$ is equal to or larger than 11. Indeed, a convergence curve has been computed in Fig. 6, showing the maximal relative error between the results obtained with a given $N$ and the results obtained with $N=25$, for several $N$ and reduced viscosity $\alpha$. As shown, the convergence is already obtained with $N=4$ for large $\alpha$. For smaller viscosities, the error becomes negligible as soon as $N>11$.

Chebyshev polynomials introduced in Eq. 29 are known to show interesting properties (Boyd 2001):

$u^{(1)} \equiv \frac{\mathrm{du}}{\mathrm{d} y}=\frac{\mathrm{d}\left(\sum \mathrm{a}_{\mathrm{k}}(\mathrm{t}) \mathrm{T}_{\mathrm{k}}(\mathrm{y})\right)}{\mathrm{d} y}=\sum a_{k}^{(1)} T_{k}(y)$, 


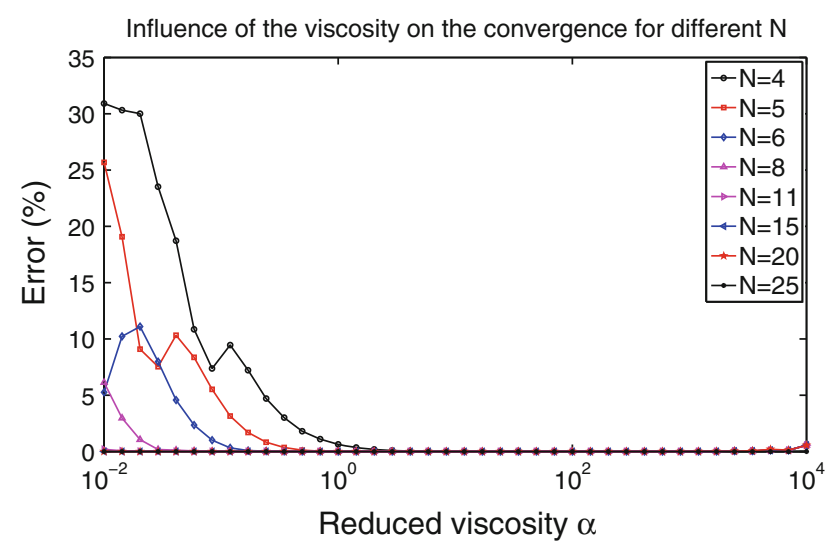

Fig. 6 Influence of the reduced viscosity $\alpha$ on the convergence curve, for different $N$

where it can be shown that the $a^{(1)}{ }_{k}$ coefficients can be expressed as:

$a_{i}^{(1)}=\sum D_{i k}^{(1)} a_{k}$,

where the $D^{(1)}$ matrix is given by:

$D_{i j^{(1)}=}$
$\begin{cases}j & \text { if } i=0 \text { and } j=1,3,5 \ldots \\ 2 j & \text { if } i=1,2, \ldots, N-1 \text { and } j=i+1, i+3, i+5 \ldots \\ 0 & \text { in the other cases }\end{cases}$

It is also known that the second derivative of the $u$ can be expressed on a similar way as:

$u^{(2)}(x)=\sum_{k=0}^{N-2} a_{k}^{(2)} T_{k}(x)$

where the $a^{(2)}{ }_{k}$ coefficients can be computed with:

$a_{k}^{(2)}=\sum D_{k i}^{(2)} a_{i}$,

where the matrix $D^{(2)}=D^{(1)} D^{(1)}$.

Consequently, the diffusion equation (23) leads to:

$\dot{u}=\sum_{k=0}^{N} \dot{a}_{k}(t) T_{k}(y) \stackrel{(23)}{=}_{=}^{\sum} a_{k}^{(2)} T_{k}$

whose identification leads to $k=0 \ldots N-2$ equations:

$\dot{a}_{k}=\alpha \sum_{j=0}^{N} D_{k j}^{(2)} a_{j}$

The boundary condition (25) allows to write $\dot{x}$ as a function of $u$ :

$\dot{x}=u(y=1)=\sum_{k=0}^{N} a_{k} \underbrace{T_{k}(1)}_{\equiv 1}=\sum_{k=0}^{N} a_{k}$ which is now used to rewrite the Newton law (22) as:

$$
\begin{aligned}
\ddot{x}{\stackrel{(37)}{=} \sum_{k=0}^{N} \dot{a}_{k} \stackrel{(}{=}^{(22)}}^{(3)}-x-\frac{\alpha}{\tilde{m}} u^{(1)}(y=1) \\
=-x-\frac{\alpha}{\tilde{m}} \sum_{k=0}^{n} a_{k}^{(1)} \underbrace{T_{k}(1)}_{\equiv 1} \\
=-x-\frac{\alpha}{\tilde{m}} \sum_{k=0}^{n} \sum_{j=0}^{n} D_{k j}^{(1)} a_{j} \\
=-x-\frac{\alpha}{\tilde{m}} \sum_{j=0}^{n}(\underbrace{\sum_{k=0}^{n} D_{k j}^{(1)}}_{\equiv f_{j}}) a_{j}
\end{aligned}
$$

Finally, the boundary condition (24) can be expressed as:

$u(y=-1)=0=\sum a_{k} T_{k}(-1)=\sum_{k=0}^{N} a_{k}(-1)^{k}$

and obviously $\dot{u}(y=-1)$ is also equal to zero, leading to: $\dot{u}(y=-1)=0$

$$
\begin{aligned}
& =\sum \dot{a}_{k} T_{k}(-1) \\
& =\sum \dot{a}_{k}(-1)^{k}+\underbrace{0}_{(39)} \\
& =\sum \dot{a}_{k}(-1)^{k}+\sum a_{k}(-1)^{k}
\end{aligned}
$$

This latter condition is rather arbitrary, but it allows to write the algebraic condition of Eq. 39 into a first order differential equation which adds an equation to the system described by Eq. 42. It can be shown that it adds an eigen value equal to -1 and an associated amplitude $w_{j}=0$ as used in Eq. 44.

Posing the unknown vector $X$ with $N+2$ unknowns defined as:

$X=\left(\begin{array}{llllll}a_{0} & a_{1} & \ldots & a_{i} & \ldots & a_{N} x\end{array}\right)^{T}$

it can be shown that the $N-1(k=0 \ldots N-2)$ Eq. 36, together with Eqs. 37, 38 and 40, constitute a $N+2$ differential equations system, which can be written as:

$M \dot{X}=A X$

with the following initial condition:

$X(t=0)=X_{0}=(\underbrace{0 \ldots 0}_{1 \times N+1} 1)^{T}$

$M$ and $A$ matrices are detailed in Tables 1 and 2. This constant coefficient system of ODEs (42) is easily solved analytically: 
Table $1 M$ matrix

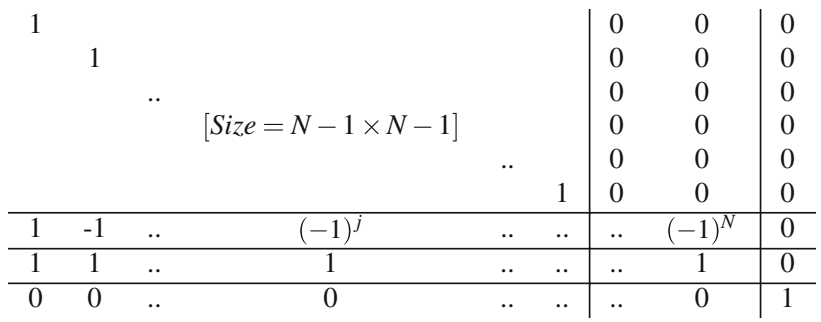

$X_{i}=\sum_{j=1}^{N+2} r_{i j} w_{j} \mathrm{e}^{\lambda_{j} t}$

where $\lambda_{j}$ is the $j$ th eigen value, $r_{i j}$ the $i$ th component of the $j$ th eigenvector associated with $\lambda_{j}$ and $w_{j}$ the $j$ th component of the vector $\mathbf{w}$ given by $\mathbf{w}=V^{-1} X_{0}$, and $V$ is the matrix whose columns are the eigenvectors $r_{j}$.

\section{Materials and methods}

The experimental setup is made of two circular pads of diameter $D$ whose separation distance $h$ can be accurately adjusted (see Fig. 7). The bottom pad is glued on a translation stage which can be displaced on a distance $e$ by a micrometric screw. The upper pad which is glued on the so-called shuttle can move according to the deformation of two parallel elastic beams of length $L$, width $b$, and thickness $t$. When both pads are linked by a liquid meniscus, the upper pad undergoes an elongation $x$ from the rest position, allowing the balance of the restoring elastic force (directed to the left on the figure) and the lateral capillary force (directed to the right of the figure) occurring from the shift $e-x$ between both pads.

The bottom pad is carried by a six dof translation stage allowing accurate alignment, tilt, and orientation. The $z$ dof tunes the gap $h$ while the $x$ dof tunes the shift. The cantilevered side of both elastic beams supporting the shuttle can be precisely moved along the $x$ - and $y$-axes.

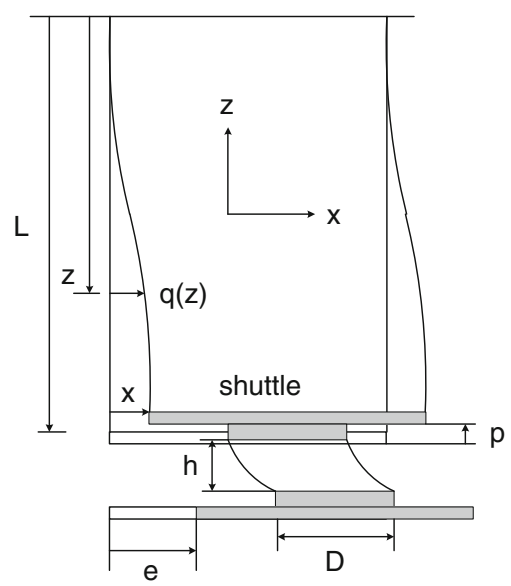

Fig. 7 Sketch of the experimental platform: a bottom pad of diameter $D$ is fixed on a translation stage which imposes to it a displacement $e$. The top pad, with the same diameter, is pulled in the direction of $x$ by the lateral capillary force to be measured and in the opposite direction by the elastic restoring force of the spring. Since there must be a balance between the restoring force $k x$ and the capillary force, we can explore the characteristic force-shift. The gap $h$ can be tuned by moving the bottom pad vertically, $p$ is a vertical parasitic motion. $q(z)$ is the deformed shape of the beam at a distance $z$ from the cantilevered side

Once the stiffness $k_{\mathrm{sh}}$ of the elastic suspension of the shuttle is known, the lateral capillary force $k_{\mathrm{sh}} e$ can be plotted as a function of the shift $e-x$ to determine the slope which is equal to the meniscus stiffness $k_{\mathrm{c}}$ from Eq. 14. The order of magnitude of the stiffness $k_{\mathrm{sh}}$ of the shuttle is $1 \mathrm{~N} \mathrm{~m}^{-1}$ and the accuracy on the micrometric screws is about $1 \mu \mathrm{m}$ leading to a force resolution of about $1 \mu \mathrm{N}$. This stiffness has been characterized on the one hand by measuring the resonance frequency and the mass of the shuttle and on the other hand by applying a calibrated horizontal force to the shuttle and measuring its elongation $x$. From these experiments, $k_{\mathrm{sh}}$ is estimated about $0.94 \mathrm{~N} \mathrm{~m}^{-1}$ by the first method and $1.06 \mathrm{~N} \mathrm{~m}^{-1}$ by the second one, leading therefore to the range $k_{\mathrm{sh}}=1 \mathrm{~N} \mathrm{~m}^{-1} \pm 0.06$.

In addition to the measurement of the static elongation from which we deduce the lateral stiffness of the meniscus, damping properties are deduced from the dynamic response

Table 2 A matrix

\begin{tabular}{cccccccc|c}
$\alpha D_{00}^{(2)}$ &.. &.. &.. &.. & $\alpha D_{0(N-2)}^{(2)}$ & $\alpha D_{0(N-1)}^{(2)}$ & $\alpha D_{0 N}^{(2)}$ & 0 \\
$\alpha D_{10}^{(2)}$ &.. &.. &.. &.. &.. &.. &.. & 0 \\
.. &.. &.. &.. &.. &.. &.. &.. & 0 \\
.. &.. &.. & $\alpha D_{i j}^{2)}$ &.. &.. &.. &.. & 0 \\
.. &.. &.. &.. &.. &.. &.. &.. & 0 \\
$\alpha D_{(N-2) 0}^{(2)}$ &.. &.. &.. &.. & $\alpha D_{(N-2)(N-2)}^{(2)}$ & $\alpha D_{(N-2)(N-1)}^{(2)}$ & $\alpha D_{(N-2) N}^{(2)}$ & 0 \\
\hline-1 & 1 &.. & $-(-1)^{j}$ &.. &.. &.. & $-(-1)^{N}$ & 0 \\
\hline.. &.. &.. & $-\frac{\alpha}{\tilde{m}} f_{j}$ &.. &.. &.. &.. & -1 \\
\hline 1 & 1 & 1 & 1 & 1 & 1 & 1 & 1 & 0
\end{tabular}




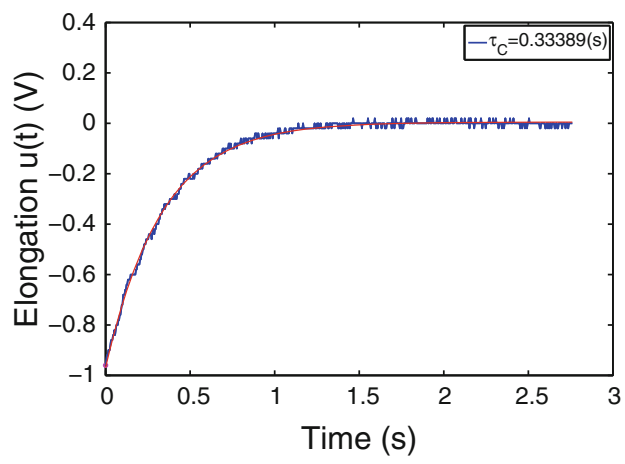

(a)

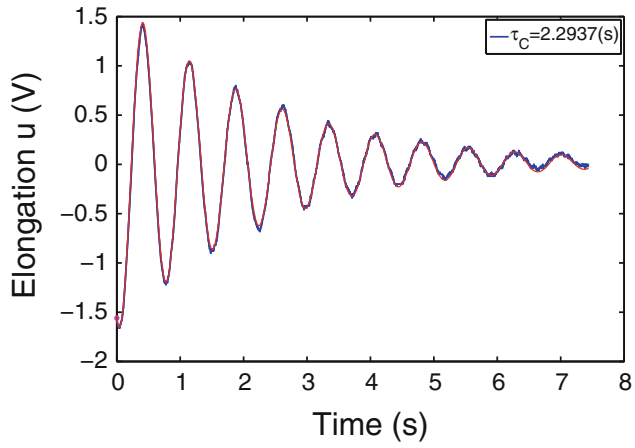

(b)

Fig. 8 Example of dynamic responses. The elongation $x(t)$ is directly given in $V$ (the voltage output is linearly proportional to the elongation $x$ in $\mathrm{m})$. Details of experiments 18 and 31 are given in Table 3. a Experiment 31 and $\mathbf{b}$ experiment 18

$x(t)$ (in this case, $e=0) . x(t)$ either shows an underdamped or an overdamped behavior as illustrated in Fig. 8 . We fit the response with either $x=A \exp \left(-t / \tau_{\mathrm{c}}\right) \cos (\omega t+\phi)+B$ or with $x(t)=A \exp \left(-t / \tau_{\mathrm{c}}\right)+B$, respectively, and hence deduce the characteristic time $\tau_{\mathrm{c}}$. Each experiment has been repeated three times, and each set of experimental data has been fitted three times, too. A signal-to-noise ratio is then built from the ratio of the average $\tau_{\mathrm{c} \text {-mean }}$ over the standard deviation $\tau_{\text {c-std }}$ (values are reported in Table 3 ).

$\tau_{\mathrm{c}}$ is then divided by the characteristic time $\tau=\sqrt{m / k}$ of Eq. 20, to produce $\tau_{\mathrm{d}}$ which is plotted in Fig. 10. Note that $m$ is here the equivalent mass of the shuttle
( $m=14.8 \mathrm{~g})$ and $k$ the sum of the experimentally deduced value of the meniscus stiffness and the stiffness of the shuttle $k_{\mathrm{sh}}=1 \mathrm{~N} \mathrm{~m}^{-1}$.

\section{Results and discussion}

\subsection{Benchmark}

We have benchmarked our method with the theoretical results of $\mathrm{Lu}$ and Bailey (2005) as indicated in Fig. 9 by superimposing the results of our work on the results of $\mathrm{Lu}$

Table 3 In this table, experiments are stacked by liquid ID (see also Table 4)

\begin{tabular}{|c|c|c|c|c|c|c|c|c|c|c|c|}
\hline \multirow{2}{*}{$\begin{array}{l}\text { Exp. } \\
n r\end{array}$} & \multirow{2}{*}{$\begin{array}{l}\text { Liquid } \\
\text { ID }\end{array}$} & \multirow{2}{*}{$\begin{array}{l}\alpha \text { no } \\
\text { unit }\end{array}$} & \multirow{2}{*}{$\begin{array}{l}\tilde{m} \text { no } \\
\text { unit }\end{array}$} & \multicolumn{6}{|l|}{ Times } & \multirow{2}{*}{$\begin{array}{l}\text { Rel. error } \\
100 \frac{\tau_{\mathrm{d}(\mathrm{sim})}-\tau_{\mathrm{d}}}{\tau_{\mathrm{d}}} \\
(\%)\end{array}$} & \multirow{2}{*}{$\begin{array}{l}\text { Oscillations Overdamped/ } \\
\text { under damped }\end{array}$} \\
\hline & & & & $\begin{array}{l}\tau_{\mathrm{c} \text {-mean }} \\
(\mathrm{s})\end{array}$ & $\begin{array}{l}\tau_{\mathrm{c}-\text { std }} \\
(\mathrm{s})\end{array}$ & $\begin{array}{l}\frac{\tau_{c-\text { mean }}}{\tau_{c-s d t}} \\
\text { unit) }\end{array}$ & $\tau(\mathrm{s})$ & $\begin{array}{l}\tau_{\mathrm{d}}=\frac{\tau_{\mathrm{c} \text {-mean }}}{\tau} \text { (no } \\
\text { unit) }\end{array}$ & $\begin{array}{l}\tau_{\mathrm{d}(\mathrm{sim})}(\text { no } \\
\text { unit) }\end{array}$ & & \\
\hline 34 & Dymax & 72.6 & 770 & 1.749 & 0.081 & 21.6 & 0.097 & 18.0 & 42.8 & 136 & Under \\
\hline 16 & 6 & 43.2 & 445 & 4.396 & 0.094 & 46.9 & 0.108 & 40.6 & 42.0 & 2 & Under \\
\hline 17 & 6 & 57.8 & 522 & 3.523 & 0.110 & 32.1 & 0.105 & 33.5 & 37.0 & 8 & Under \\
\hline 18 & 6 & 90.8 & 661 & 2.306 & 0.138 & 16.7 & 0.103 & 22.4 & 29.7 & 30 & Under \\
\hline 19 & 6 & 119 & 777 & 2.147 & 0.153 & 14.0 & 0.098 & 21.9 & 26.6 & 19 & Under \\
\hline 10 & 1 & 395 & 597 & 0.686 & 0.070 & 9.8 & 0.107 & 6.3 & 6.1 & -3 & Under \\
\hline 8 & 7 & 761 & 593 & 0.272 & 0.012 & 23.1 & 0.104 & 2.6 & 3.2 & 20 & Under \\
\hline 9 & 7 & 2,390 & 1,030 & 0.101 & 0.013 & 7.7 & 0.110 & 0.9 & 1.7 & 92 & Over \\
\hline 26 & 7 & 451 & 440 & 0.475 & 0.010 & 48.0 & 0.113 & 4.2 & 4.0 & -7 & Under \\
\hline 27 & 7 & 669 & 552 & 0.366 & 0.007 & 50.9 & 0.106 & 3.4 & 3.4 & -3 & Under \\
\hline 28 & 7 & 1,580 & 855 & 0.236 & 0.009 & 26.8 & 0.104 & 2.2 & 2.2 & -2 & Under \\
\hline 3 & 2 & 1,700 & 386 & 0.254 & 0.029 & 8.6 & 0.110 & 2.3 & 1.6 & -32 & Over \\
\hline 29 & 2 & 2,190 & 439 & 0.193 & 0.010 & 20.2 & 0.109 & 1.7 & 2.0 & 17 & Over \\
\hline 30 & 2 & 2,610 & 484 & 0.249 & 0.008 & 29.8 & 0.107 & 2.3 & 2.3 & -2 & Over \\
\hline 31 & 2 & 4,030 & 609 & 0.305 & 0.024 & 12.6 & 0.104 & 2.9 & 3.0 & 2 & Over \\
\hline
\end{tabular}

The characteristic time $\tau_{\mathrm{c}}$ is the characteristic time obtained from the dynamic response of the shuttle $x(t)$. As indicated in the text, these data have been averaged $\left(\tau_{\mathrm{c} \text {-mean }}\right)$ and the standard deviation $\tau_{\text {c-std }}$ has been used to compute a signal-to-noise ratio, which is shown to be always larger than 7.7. The characteristic time $\tau=\sqrt{m / k_{\mathrm{d}}}$ is recalled--where $m$ is the equivalent mass of the shuttle and $k_{\mathrm{d}}$ is the sum of the shuttle stiffness $k=1 \mathrm{~N} \mathrm{~m}^{-1}$ and the meniscus stiffness measured in that experiment (not shown here). The column gives $\tau_{\mathrm{d}}$, the non-dimensional ratio of $\tau_{\mathrm{c}}$ over $\tau$, which is plotted in Fig. 10. $\tau_{\mathrm{d}}$ can be compared with the simulated $\tau_{\mathrm{d}(\mathrm{sim})}$. The last column indicates the experimental damping behavior 


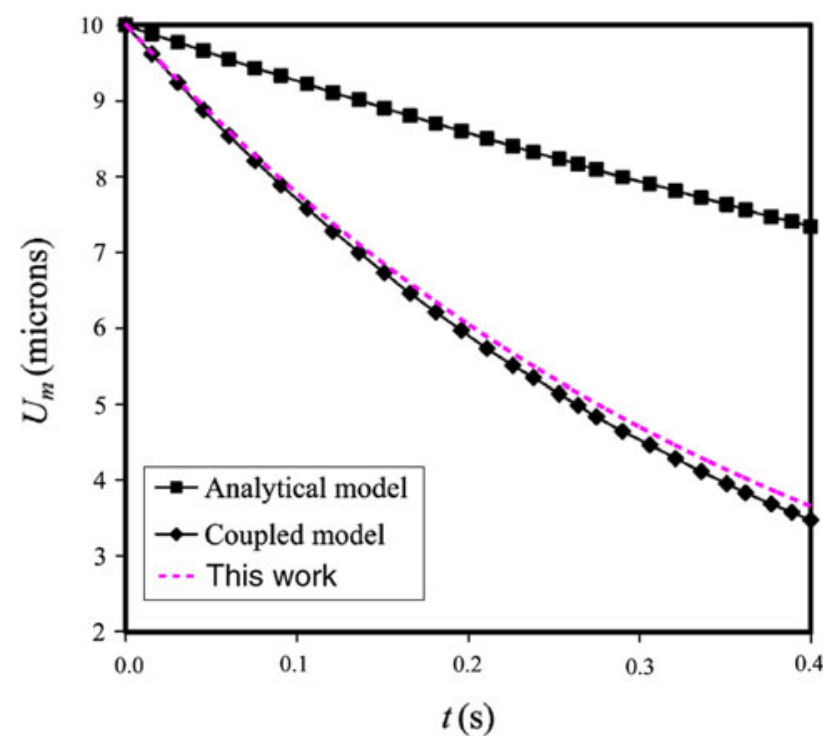

Fig. 9 Benchmark of our solution by comparison with Fig. 9 from $\mathrm{Lu}$ and Bailey. For $\alpha=0.18, \tilde{m}=99, \delta=10 \mu \mathrm{m}, N=11$, we found a non dimensional damping time $\tau_{\mathrm{d}}=0.3958$, where Lu and Bailey found $\tau_{\mathrm{d}}=0.3784$ (4\% difference)

and Bailey (Fig. 9 of their article). The difference is about $4 \%$. The main advantage of the proposed resolution method is to reduce the computing time drastically while still keeping the physics coupled (224 couples $(\tilde{m}, \alpha)$ can be computed in 5-10 $\mathrm{min}$ ), by comparison with the method of Lu and Bailey using Surface Evolver and a CFD package for the flow problem. This advantage permits the parametric study presented in the next subsection.

\subsection{Numerical results}

For any couple of parameters $(\alpha, \tilde{m})$, Eq. 44 gives $x(t)=X_{N+2}(t)$ the position of the plate as a combination of different modes whose amplitude decreases with time (real parts of $\lambda_{j}$ are negative). Consequently, the decrease of $x$ with time is governed by the slowest mode, i.e., by the mode with the eigen value $\lambda$ whose (negative) real part has the smallest absolute value. $\tau_{\mathrm{c}}$ is then equal to $-1 / \lambda$. The combinatory space defined by $\alpha=40-4,000$ (logspace with 16 points) and $\tilde{m}=400-1,000$ (logspace with 14 points) has been explored, outputing a grid of $16 \times 14$ points. The $\tau_{\mathrm{c}}$ at these points have been divided by their respective characteristic time $\tau=\sqrt{m / k}$ and interpolated with Matlab using the contour function in order to display the iso-lines of Fig. 10.

\subsection{Experimental results}

A large set of experiments has been performed, as indicated in Table 3, whose column "liquid ID" points to the liquid properties given in Table 4. The good signal-to-

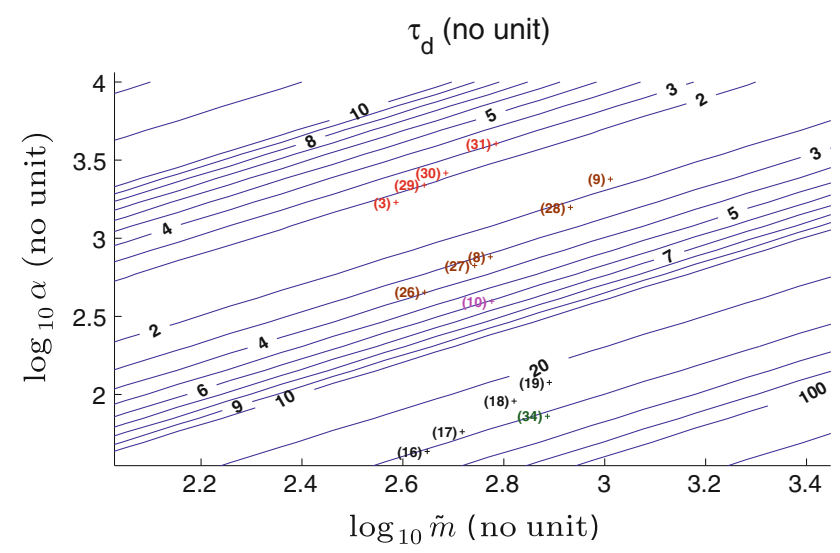

Fig. 10 Non-dimensional map of damping times, for experiments 3, 29-31 (Oil 2); 10 (Oil 1); 16-19 (Oil 6); 8-9, 26-28 (Oil 7); 34 (Dymax $628 \mathrm{VLV})$. The family set of parallel lines represents the isovalues of $\tau_{\mathrm{d}(\mathrm{sim})}$ obtained by simulation. Each experiment number is indicated between brackets, the '+' mark to its right showing the exact location of the experimental couple $(\tilde{m}, \alpha)$. The numerical comparison between simulation and experiment can be found in Table 3, this map of iso-values of $\tau_{\mathrm{d}}$ can be used to estimate the damping time. For example, experiment $30(\alpha=2,610, \tilde{m}=484)$ lies between the isoline 2 and the iso-line 3 (the measured value is 2.3 )

noise ratio given by the ratio of the average over the standard deviation of characteristic time $\tau_{\mathrm{c}}$ is reported. Missing numbers in the table are experiments that were discarded because of smaller signal-to-noise ratio, which would have led to a large scattering of results; they correspond to less viscous liquids, i.e., water $(\eta=1 \mathrm{mPa} \mathrm{s})$ and Dow Corning DC200FLUID10 oil $(\eta=9.3 \mathrm{mPa} \mathrm{s})$, leading to longer damping times up to $32 \mathrm{~s}$ : since $u(t)$ has not been registered on a sufficiently large period of time, the amplitude decrease was not large enough to ensure reliable fit, hence a larger standard deviation. For the other experiments, we can see in Fig. 10 the fair concordance between simulation and experimental results. Thanks to both non-dimensional parameters $\alpha$ and $\tilde{m}$, the map of Fig. 10 contains all information concerning the dynamical lateral behavior of a liquid meniscus.

\subsection{Analytical expression of the damping time}

In addition, it is worth noting that in Fig. $10, \tau_{\mathrm{d}}$ contour lines are essentially straight lines of the form:

$\log _{10} \alpha-\log _{10} \alpha_{0}=\beta\left(\log _{10} \tilde{m}-\log _{10} \tilde{m}_{0}\left(\tau_{\mathrm{d}}\right)\right)$

with $\beta=1.0241, \log \alpha_{0}=4$ and the relationship between $\tilde{m}_{0}$ and $\tau_{\mathrm{d}}$ being expressed as:

$\log _{10} \tau_{\mathrm{d}}=a+b \log _{10} \tilde{m}_{0}$

where $(a, b)=(3.7,-1)$ if $\log _{10}(\alpha-4)>\beta\left(\log _{10} \tilde{m}-\right.$ $3.55)$ else $(a, b)=(-3.4,1)$ (Fig. 11). Consequently, the non-dimensional characteristic time $\tau_{\mathrm{d}}$ of Fig. 10 can be estimated by the following expression in case $\alpha>10$ : 
Table 4 Liquid properties

\begin{tabular}{lclll}
\hline Liquid ID & $\rho\left(\mathrm{kg} \mathrm{m}^{-3}\right)$ & $\eta(\mathrm{Pa} \mathrm{s})$ & $\gamma\left(\mathrm{N} \mathrm{m}^{-1}\right)$ & Supplier \\
\hline 6 & 960 & 0.096 & 0.0209 & Dow Corning DC200FLUID100 \\
1 & 970 & 0.485 & 0.0211 & Rhodorsil R47V500 \\
7 & 971 & 0.971 & 0.0212 & Dow Corning DC200FLUID1000 \\
2 & 973 & 4.865 & 0.0211 & Rhodorsil R47V5000 \\
Dymax & 1,050 & 0.055 & 0.025 & $\rho$ and $\eta:$ Dymax 628-VLV data \\
& & & & $\gamma:$ own measurement \\
\hline
\end{tabular}

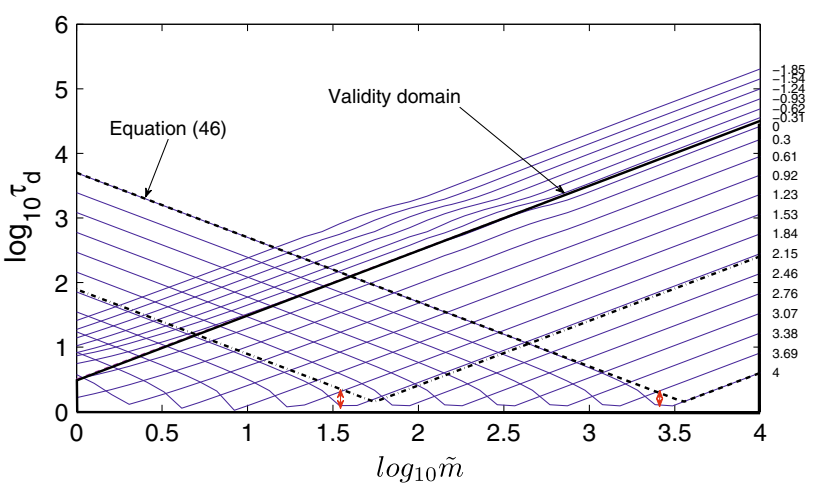

Fig. 11 This figure graphically illustrates the evolution of the characteristic damping time $\tau_{\mathrm{d}}$ of Fig. 10 as a function of $\tilde{m}$ for different values of $\alpha$ (the small figures to the right of this plot indicate the values $\log _{10} \alpha$ ). It can be seen that these values can reasonabily be estimated by straight lines (the dashed line is given for $\alpha=10,000$, whose equation is given by Eq. 46). The coefficients $a$ and $b$ associated to Eq. 46 are valid for $\log _{10} \alpha$ larger than 0 . This simplified model suffers from a small lack of fit indicated by red double arrows. It is however shown with the dashed-dotted line the fair concordance, at least within the validity domain indicated by the bold black contour

$\log _{10} \tau_{\mathrm{d}}=b \log _{10} \tilde{m}-\frac{b}{\beta} \log _{10} \alpha+a+\frac{4 b}{\beta}$

\subsection{Over- and underdamping}

Theoretically, a mechanical system governed by Eq. 1 is underdamped if $c^{2}-4 k m<0$. Since $c$ is related to the dimensional characteristic time $\tau_{\mathrm{c}}$ by the relation $c=2 \mathrm{~m} /$ $\tau_{\mathrm{c}}$, the former condition for overdamping becomes $\tau_{\mathrm{c}}>\sqrt{\frac{m}{k}}$ which can also expressed using the non-dimensional damping time $\tau_{\mathrm{d}}$ as $\tau_{\mathrm{d}}>1$. Experimentally, the last column of Table 3 indicates a border between both behaviors between 2 and 3 instead of 1 .

\section{Conclusions and future work}

We showed that the coupled problem of liquid and chip dynamics could be efficiently solved with a pseudo-spectral method based on Chebyshev polynomials. The theoretical results were benchmarked using literature results, and a thorough experimental validation was pursued. Some discrepancies were, however, observed for low viscosity configurations (i.e., low values of the non-dimensional parameter $\alpha$ ). These discrepancies are thought to be caused by a too-short acquisition time of the position $u(t)$ of the chip, leading to a non-robust fitting and a characteristic time with large scattering. Therefore, for various experiments covering the experimental space $\alpha=40-4,000$ and $\tilde{m}=400-1,000$, the numerical proposed resolution and the subsequent modeling have been proven reliable. From the linear relation between $\alpha$ and $\tilde{m}$, we could propose a mathematical formulation of the damping time.

Future experiments will try to fix the scattering problem for low $\alpha$ experiments. Future work will consist in developing a full resolution of the liquid flow between the pad and the chip, in order to determine the limits of the spectral resolution. Finally, it could be interesting to develop two new experimental setups: the first one would include a heat source in order to work with actual solder pastes and the second one would be downsized in order to tackle the $10-100 \mu \mathrm{m}$ scale.

Acknowledgements This work is conducted with financial support from the project Hybrid Ultra Precision Manufacturing Process Based on Positional and Self assembly for Complex Micro-Products (HYDROMEL NMP2-CT-2006-026622) funded by the European Commission. A special thanks to B. Tartini.

\section{References}

Abbasi S, Zhou AX, Baskaran R, Bohringer KF (2008) Part tilting in capillary-based self-assembly: modeling and correction methods. In: Proceedings of the IEEE 2008 conference on MEMS Tucson (AZ), January 13-17

Avital A, Zussman E (2006) Fluidic assembly of optical components. Trans IEEE Adv Packag 29(4):719-724

Bohringer KF, Liang S-H, Xiong X (2004) Towards optimal designs for self-alignment in surface tension driven self-assembly. In: Proceedings of the 17th IEEE international conference on MEMS, IEEE, pp 9-12

Boufercha N, Sägebarth J, Burgard M, Othman N, Schlenker D, Schäfer W, Sandmaier H (2008). Micro-assembly with fluids. MST/NEWS 2/08:29-30

Boyd JP (2001) Chebyshev and Fourier spectral methods. Dover Publications, Inc., New York

Brakke K (1992) The surface evolver. Exp Math 1(2):141-165 
Engmann J, Servais C, Burbidge AS (2005) Squeeze flow theory and applications to rheometry: a review. J Non-Newton Fluid Mech 132(1-3): $1-27$

Fang J (2006) Self-assembly techniques for massively parallel packaging of MEMS devices. PhD thesis, University of Washington, Seattle

Hong YK, Syms RRA (2006) Stability of surface tension selfassembled 3d moems. Sens Actuators A 127:381-391

Kaneda M, Yamamoto M, Nakaso K, Yamamoto T, Fukai J (2007) Oscillation of a tilted circular pad on a droplet for the selfalignment process. Precis Eng 31:177-184

Kim JM, Shin YE, Fujimoto K (2004) Dynamic modeling for resin self-alignment mechanism. Microelectron Reliab 44:983-992

Lambert P (2007) Capillary forces in microassembly: modeling, simulation, experiments, and case study. Microtechnology and MEMS. Springer, New York

Lin W, Patra SK, Lee YC (1995) Design of solder joints for selfaligned optoelectronic assemblies. IEEE Trans Adv Packag 18(3):543-551

Lin C, Tseng F, Kan HC, Chieng C-C (2009) Numerical studies on micropart self-alignment using surface tension forces. Microfluid Nanofluid 6(1):63-75

Lu H, Bailey C (2005) Dynamic analysis of flip-chip self alignment. IEEE Trans Adv Packag 28(3):475-480

Mastrangeli M, Abbasi S, Varel C, Van Hoof C, Celis J-P, Bohringer KF (2009a). Self-assembly from milli- to nanoscales: methods and applications. J Micromech Microeng 19:1-37

Mastrangeli M, Ruythooren W, Van Hoof C, Celis J-P (2009b) Conformal dip-coating of patterned surfaces for capillary die-tosubstrate self-assembly. J Micromech Microeng 19(4):12

Meurisse M-H, Querry M (2006) Squeeze effects in a flat liquid bridge between parallel solid surfaces. J Tribol 128(3):575-584
Onoe H, Matsumoto K, Shimoyama I (2007). Three-dimensional sequential self-assembly of microscale objects. Small 3(8):13831389

Park S, Bohringer KF (2008) A fully dry self-assembly process with proper in-plane orientation. In: Proceedings of the IEEE 2008 Conference on MEMS

Pascarella NW, Baldwin DF (1998) Compression flow modeling of underfill encapsulants for low cost flip chip assembly. In: Proceedings of the 1998 international symposium of underfill encapsulants for low cost flip chip assembly, p 33

Saeedi E, Abbasi S, Bohringer KF, Parviz BA (2006) Molten-alloy driven self-assembly for nano and micro-scale system integration. FDMP 2(4):221-245

Sariola V, Zhou Q, Laass R, Koivo HN (2008) Experimental study on droplet based hybrid microhandling using high speed camera. In: Proceedings of the 2008 IEEE/RSJ international conference on intelligent robots and systems, pp 919-924, Nice, September $22-26$

Tsai CG, Hsieh CM, Yeh JA (2007) Self-alignment of microchips using surface tension and solid edge. Sens Actuators A 139: 343-349

van Veen N (1999) Analytical derivation of the self-alignment motion of flip chip soldered components. J Electron Packag 121:116121

Zhang X, Chen C-C, Bernstein RW, Zappe S, Scott MP, Solgaard O (2005) Microoptical characterization and modeling of positioning forces on drosophila embryos self-assembled in twodimensional arrays. J Microelectromech Syst 14(5):1187-1197

Zheng W, Buhlmann P, Jacobs HO (2004) Sequential shape and solder directed self-assembly of functional microsystems. PNAS 101(35):12814-12817 